\title{
A Canonical 3-D P53 Network Model that Determines Cell Fate by Counting Pulses
}

\author{
Gökhan Demirkıran' ${ }^{1}$, Güleser Kalaycı Demir² ${ }^{\text {iD }}$, Cüneyt Güzeliş ${ }^{1}$ \\ 'Department of Electrical and Electronics Engineering, Yaşar University School of Engineering, İzmir, Turkey \\ 2Department of Electrical and Electronics Engineering, Dokuz Eylül University School of Engineering, İzmir, Turkey
}

Cite this article as: G. Demirkıran, G. Kalaycı Demir, C. Güzeliş, "A Canonical 3-D P53 Network Model that Determines Cell Fate by Counting Pulses", Electrica, vol. 18, no: 2, pp. 284-291, 2018.

\section{ABSTRACT}

From a system theory perspective, p53 network dynamics is interesting since it can exhibit three dynamical modes of p53, namely low-level equilibrium, oscillation, and high-level equilibrium. Each of these modes are associated with different cell fate outcomes: cell survival, cell cycle arrest, and apoptosis. The literature reveals that a high level (apoptosis) is seen only after ending the oscillation phase, so called two-phase dynamics, which provides the decision of apoptosis depending on the oscillation duration. This paper proposes that a negative feedback can keep time by counting the pulses of oscillation to take the decision of apoptosis or cell survival. P53DINP1, which is the mediator of this feedback, is added as a variable to a 2-D oscillator model of the p53 network. The resulting canonical 3-D model successfully replicates the two-phase dynamics. That is, it possesses temporary oscillatory behavior, in which first oscillations (first phase) and then high level state (second phase) are observed. By introducing a new variable to the core oscillator in the p53 network, this study demonstrates that p53 network can be considered a modular structure, which consists of an oscillator and other variables that control this oscillator to contribute to cell fate determination.

Keywords: Oscillators, p53 network, two-phase dynamics, gene regulatory networks, cell fate, P53DINP1

\section{Corresponding Author:}

Gökhan Demirkıran

\section{E-mail:}

gokhan.demirkiran@yasar.edu.tr

Received: 06.03 .2018

Accepted: 15.05 .2018

(c) Copyright 2018 by Electrica

Available online at

http://electrica.istanbul.edu.tr

DOI: 10.26650/electrica.2018.02664

\section{Introduction}

P53 gene regulatory network responds to Double Strand Breaks (the most deleterious type of DNA damage) by regulating the necessary biological processes such as DNA repair, cell cycle arrest (i.e. the cell stops growing to avoid passing the damaged DNA to baby cells) and apoptosis (i.e. programmed cell death) [1]. Experiments show that these processes are associated with different dynamical modes of p53 concentration level. More specifically:

i) Low level of p53 indicates that there is no DNA damage in the cell so the cell can continue its normal cell cycle progression [2]. In other words, the cell may eventually proliferate since there is no DNA damage.

ii) Oscillation of p53 level is the indicator of DSBs in DNA [3-6]. It has been shown that oscillation of p53 level helps to repair DSBs and also causes cell cycle arrest [7]. The oscillation of p53 lasts until the DSBs are repaired, and goes to low level after the repair. However, if the repair takes too long, then the dynamical mode of p53 switches from the oscillation to a high level [8].

iii) High level of p53 triggers apoptosis, thus removing the irreparable cell from the organism [9-11].

To regulate the response to DSBs, there are three different dynamical modes (low level equilibrium, oscillations, and high level equilibrium) and three critical mode transitions. The first transition is from low level to oscillations upon the formation of DSBs; the second transition is from oscillations to low level upon the repair of DSBs; and the third transition is from oscillations to high level if the repair takes too long. According to discussions in the literature, there is no direct transition from low level to high level of p53. In other words, p53 level goes to high level 
only after an oscillatory phase. This mechanism is considered to be useful due to the fact that it will try to repair DNA damage giving the cell a chance of surviving before taking the decision of apoptosis $[3,8]$. In the literature several oscillator models have been proposed based on ATM inhibition of p53 inhibitor Mdm2 $[3,6]$. Models based on the feedback loop interaction between ATM and Wip1 dynamics have also been proposed in keeping with experimental studies demonstrating that coaction of ATM and Wip1 is crucial in the regulation of cell cycle checkpoints and apoptosis [6, 12-15].

Although oscillatory dynamics of p53 alone have been studied extensively, the study of three dynamical modes of p53 in the same model is restricted to only a few studies [16]. The study in [17] proposes a model that exhibits these modes in a two-phase dynamics fashion in which the high level of p53 (the second phase) is observed only after the oscillation of p53 (the first phase). In the model by [17], the duration of DSB repair activity determines if there will be a mode transition from the oscillations to high level of p53. In this sense, the model of [17] understands the duration of DSB repair activity by a pulse counting mechanism which counts the pulses of oscillation. If the number of pulses passes a certain value while the repairing process continues, then the decision of apoptosis is made. If the DSBs are repaired before a certain number of pulses are exhibited, then p53 goes to low level and the cell survives.

Although the model by successfully shows these three distinct modes, the intuitive understanding of the model is very hard since the model is 17 dimensional and has too many tuned parameters [17]. Recently, Demirkıran et al. [18] successfully reduced the two-phase dynamics model by into a 2-dimensional model with rational terms, which is capable of showing these three distinct modes when the critical parameters of the model are changed externally [17, 18]. The reduced 2-D model is shown to be a relaxation oscillator model which enhances the understanding of oscillations as well making corrections on the interpretation of the original 17-D model [19].

Although the reduced 2-D model with rational terms has provided a greater depth of understanding, the terms of the model are too complex [19]. Thus, Demirkıran et al. [18] proposed a simple canonical 2-D model of polynomial type, whose parameters are interpreted from p53 network interactions [20]. This simple canonical 2-D model is also capable of exhibiting 3 distinct modes when the critical parameters of the model are changed externally in the solver algorithm. In the study presented by this paper, we have extended the model in [20] by introducing new variables that automatically manipulate those critical parameters, so that a response in a two-phase dynamics fashion can be obtained. The main goal of this paper is to provide mechanistic insights into the two-phase dynamics and into the pulse counting mechanism. In this regard, our contributions in this paper are as follows:
- We propose a simple 3-D model extending the canonical 2-D model in [20], which makes the decision of apoptosis by a pulse counting mechanism.

- The 3-D model which we introduce suggests a new minimal motif as a decision block for gene regulatory networks.

- We show that a simple negative feedback (not necessarily a bistable hysteretic switch) can also cause a switching action, thus suggesting a new Oscillation Accumulation Triggered Genetic Switch (OATGS) mechanism.

The outline of the paper is as follows. In Section 2, we introduce the model and how new parameters can be tuned by giving mechanistic insights. In Section 3, we discuss that the introduced model is a new compact motif with the property of coincidence detection. In Section 4, we conclude with an emphasis on the modular perspective of p53 network.

\section{Model Description}

The schematic illustration of the interaction between the components of the model are illustrated in Figure 1. P53DINP1 was introduced as a new component to the ATM*-Wip1 system in [20] to inform ATM*-Wip1 system about the duration of DSB repair activity. The working mechanism of the resulting system is as follows. When there are DSBs in DNA, the repair molecules form complexes with DSBs, so-called DSB-complexes (DSBCs), until the repair finishes. These complexes act as external stimuli that excite the system such that $\left[\right.$ ATM $^{*}$ ] (active ATM level) and [Wip1] oscillate. As [ATM*] oscillates, it promotes the accumulation of P53DINP1 protein, which is revealed as an accumulating component in two-phase dynamics model in another paper [19]. If DSBCs disappear, meaning that DSBs are repaired, then the ATM level goes down. If DSBCs persist long enough then P53DINP1 accumulates to a certain threshold level that cuts off Wip1 feedback loop as illustrated in Figure 1. Thus, in contrast to the model in [20], the proposed model in this study responds to DSBs in a two-phase dynamics fashion by considering the duration of oscillations via a pulse counting mechanism. This is not in keeping with the study conducted in [20] in which the response is obtained by manipulating the parameters externally in the solver.

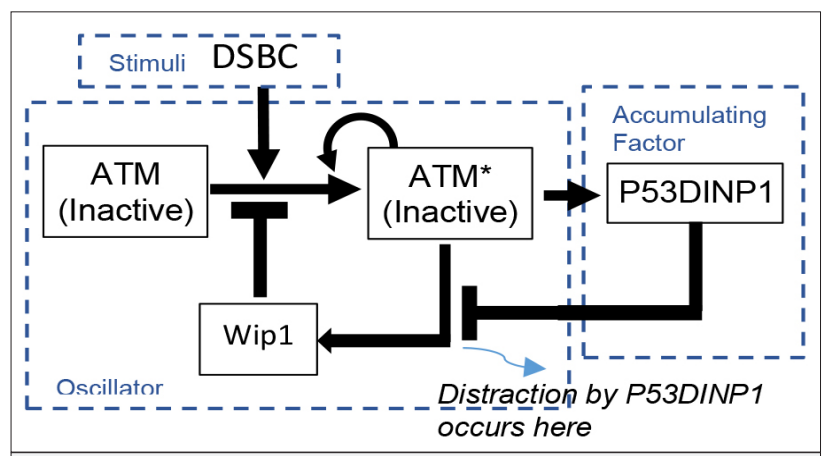

Figure 1. The schematic illustration of the interaction among the model components 
Table 1. The model equations

\begin{tabular}{|c|c|}
\hline $\begin{aligned} \frac{d\left[A T M^{*}\right]}{d t}=-[ & \left.A T M^{*}\right]\left(r\left(\left[A T M^{*}\right]^{2}\right.\right. \\
& \left.-a\left[A T M^{*}\right]-d\right)+b \\
& +c[\text { Wip } 1])\end{aligned}$ & (1) \\
\hline$d[W i p 1]$ & \\
\hline $\begin{aligned} & d t \\
= & z+m_{f}([P 53 D I N P 1])\left[A T M^{*}\right] \\
- & n[\text { Wip } 1]\end{aligned}$ & (2) \\
\hline $\begin{aligned} \frac{d[P 53 D I N P 1]}{d t} & =\varepsilon\left(\left[A T M^{*}\right]\right. \\
& -\beta[P 53 D I N P 1])\end{aligned}$ & (3) \\
\hline $\begin{array}{l}\text { where } \\
\qquad m_{f}([P 53 D I N P 1]) \\
\quad=1.5 \frac{0.6}{[P 53 D I N P 1]+0.6}\end{array}$ & (4) \\
\hline
\end{tabular}

In the proposed model (Table 1), [ATM*], [Wip1] and [P53DINP1] represent the concentrations of the corresponding proteins. In (1)-(4), ATM protein has auto-activation property modelled by the terms "rd[ATM*]" and a[ATM*]^2. This auto-activation property is responsible for the rapid activation of ATM [21, 22]. The term "-b[ATM*]" is for the self-degradation. The term "-r $\left[\text { ATM }^{*}\right]^{3 \prime}$ is needed to obtain the bistability property of ATM $[21,23]$, which can be obtained from a $3^{\text {rd }}$ order polynomial $[24,25]$ and also stays for the self-degradation of ATM. Wip1 is known to be a strong inhibitor of ATM [12]. Therefore, the term "-c[ATM*][Wip1]" is included to represent the deactivation property of Wip1.

[Wip1] is regulated positively by ATM with the term " $m_{f}[P$ 53DINP1][ATM]" where $m_{f}([P 53 D I N P 1])$ is a function with positive values as given in (4). Furthermore, [Wip1] feedback loop model (Equation (2) of Table 1) is constructed with the constant production term " $z$ ", a self-degradation term " $n$ ", following the steps of [20] in modelling Wip1 feedback loop. Not in keeping with [20], the constant parameter is replaced by (4) to take P53DINP1 dynamics into account. Also, a new variable [P53DINP1], new parameters $\varepsilon, \beta$ and a new scalar repressor function $\mathrm{m}_{\mathrm{f}}([P 53 D I N P 1])$ are introduced as detailed below.

The model variables are [ATM*], [Wip1], and [P53DINP1]. Equations (1)-(2) are taken from [20], with one exception, namely that the constant parameter $m$ is replaced by the function $\mathrm{m}_{\mathrm{f}}(P 53 \mathrm{DINP1})$. The model in [20] was based on the interaction between ATM $^{*}$ and Wip1 isolated from P53DINP1 effect. The model was capable of exhibiting three distinct modes, namely low level equilibrium, oscillations and high level equilibrium. Following a minimalist approach, the model in [20] was designed not to have p53 as a variable since ATM is the upstream mediator of p53 and oscillations of p53 result from oscillations of ATM as detailed in the references [18-20]. Thus, [ATM*] was chosen as the representative variable of p53 network dynamics. In our study, we follow the same approach again. Thus, in this minimal approach of modelling p53 network dynamics, p53 is not introduced as a dynamic variable but assumed to follow ATM dynamics.

To model the distraction of Wip1 feedback loop by P53DINP1, $\mathrm{m}_{\mathrm{f}}([\mathrm{P53DINP1])}$ must be in reverse proportion with the level of [P53DINP1]. Thus, we choose the function as in (4). Although more complex kinetic terms may be introduced, we choose a simple yet effective function that models the repressing effect.

The parameters $\varepsilon$ and $\beta$ determine the accumulation characteristics of [P53DINP1] as will be detailed in Section 2.1. In (3), $\left[\right.$ ATM $\left.^{*}\right]$ enhances $[P 53 D I N P 1]$. The parameters $\varepsilon$ and $\beta$ are chosen such that [P53DINP1] is enhanced quickly but decays slowly to model its accumulation over oscillations. The parameter values are $a=5, b=10, c=15, d=70, z=0.5, m=1.25, n=0.8$, $\varepsilon=0.02, \beta=1$ and the parameter $r$ is an external stimulus indicating the status of DSBC activity. $r=1$ means there is a full DSBC activity whilst $r=0$ means there is no DSBC activity (i.e. no DSBs in DNA). These values of the parameters will be used throughout the paper unless stated otherwise. The numerical simulation of the model demonstrating the two-phase dynamics and accumulation of [P53DINP1] is shown in Figure 2. In the time interval $[0,10]$, there is no DSBC activity indicated with $r$ $=0$. In the interval $[10,20]$, there is a short duration of DSBC activity which stimulates the oscillations. In the interval [20, 60], there is no DSB so $r=0$ and $\left[\right.$ ATM $\left.^{*}\right]$ is at low level. In a long time interval of $[60,120]$, there is DSBC activity. In this interval, oscillations are first observed (in $[60,75]$ ) and after four pulses [ATM*] level goes to a high level indicating apoptosis.

The change in [P53DINP1] can be observed as in Figure 2. It accumulates over oscillations and distracts Wip1 feedback loop when its level passes above a certain threshold level, which is around 1. Thus, [P53DINP1] functions as a switch when its level passes above a certain threshold level.

\section{Tuning the Parameters for P53DINP1}

Equation (3) is a simple example of a negative autoregulation observed in gene regulatory networks [26]. The change of [P53DINP1], i.e. derivative, is equal to the difference between its production due to $\left[\mathrm{ATM}^{*}\right]$ and self-degradation. To investigate (3) better with the meaning of parameters $\varepsilon$ and $\beta$, we take $\left[\mathrm{ATM}^{*}\right]$ as constant at its two extreme values: the maximum and minimum peaks of the [ATM*] pulses which are around 15 and zero, respectively. In the constant $\left[\mathrm{ATM}^{*}\right]$ case, the solution to (3) is as below:

$[P 53 D I N P 1]=S_{S S}+\left(S_{0}-S_{s S}\right) e^{-\varepsilon \beta t}$

Where $S_{S S}=\left[\mathrm{ATM}^{*}\right]_{\text {constant }} / \beta$ indicating the steady state value of $\left[\right.$ P53DINP1], $\mathrm{S}_{0}$ is the initial condition and $\left[\mathrm{ATM}^{*}\right]_{\text {constant }}$ is one of 


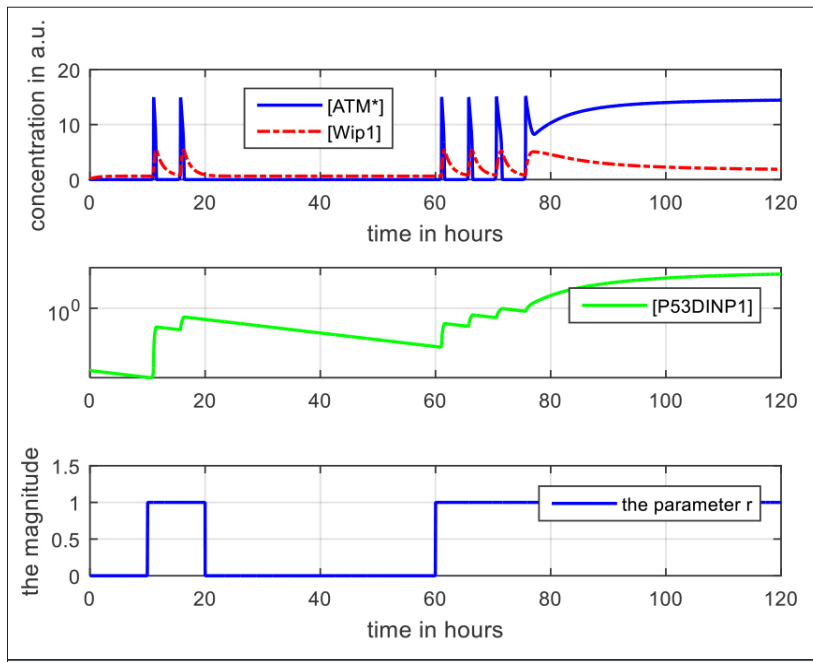

Figure 2. Demonstration of two-phase dynamics and pulse counting mechanism by the proposed 3-D model in Table 1. (Note that a.u. stands for arbitrary unit)

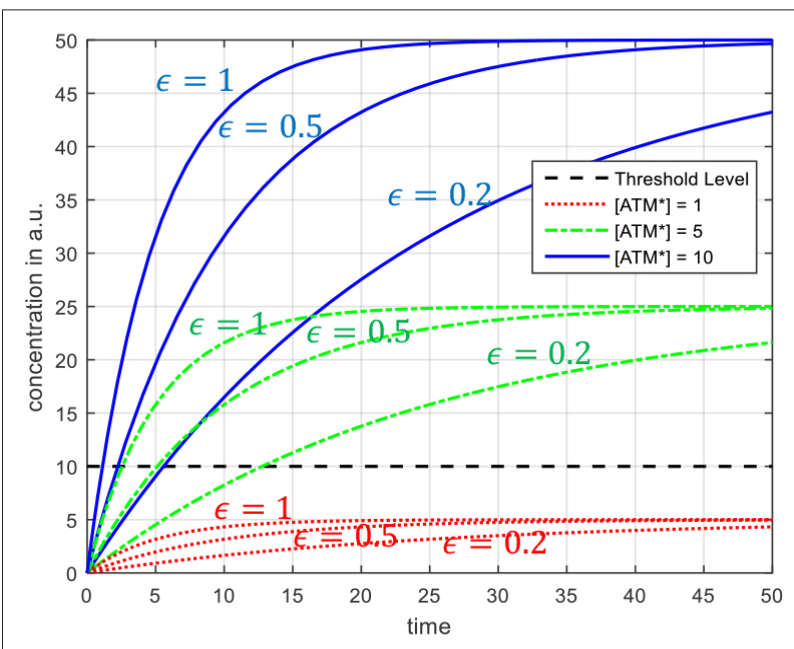

Figure 3. $[\mathrm{P53DINP1}]$ trajectories of (5) for different $\left[\mathrm{ATM}^{*}\right]$ and $\varepsilon$ values. $\beta=1$

the two extreme values of $\left[\right.$ ATM $\left.^{*}\right]$. So $\left[\text { ATM }^{*}\right]_{\text {constant }}$ and $\beta$ determine the steady state of [P53DINP1]. The product of the parameters $\varepsilon$ and $\beta$ appears as a time constant. Thus, the product $\varepsilon \beta$ determines how fast [P53DINP1] will decay. Also, it determines how fast [P53DINP1] will increase. The reason we introduce two parameters $\varepsilon$ and $\beta$ as in (3) is to give the flexibility of control over the steady state and the response time of [P53DINP1] separately when tuning the parameters.

As illustrated in Figure 3, we numerically solve (3) for different $\left[\right.$ ATM $\left.^{*}\right]$ and $\varepsilon$ values with an initial condition of [P53DINP1] $=0$ when $\beta=1$. To make our point we assume a hypothetical threshold value of 10 . As can be seen, the constant $\left[\right.$ ATM $\left.^{*}\right]$ value has effect on the steady state level of [P53DINP1]. This implies that a large [ATM*] may speed up

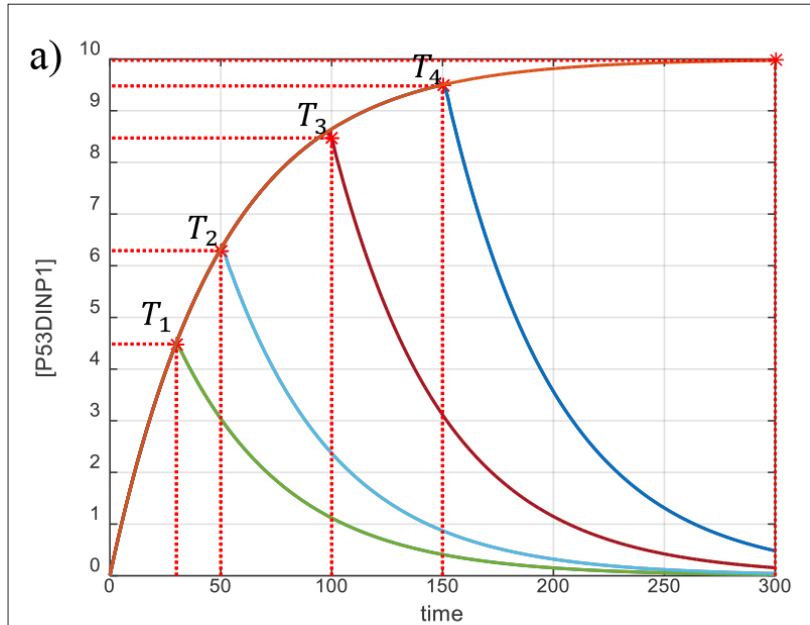

b)

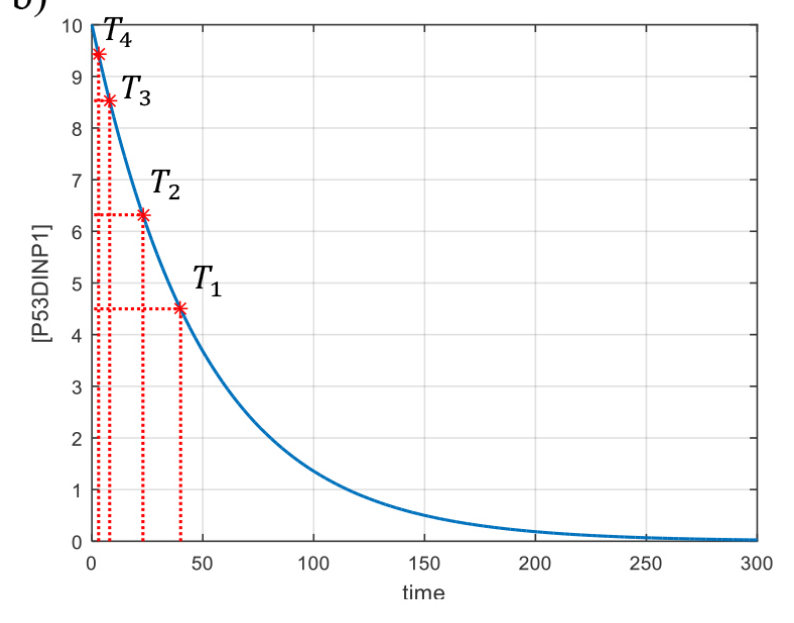

Figure 4. a, b. Time to reach to arbitrary states (a) and time to decay from those arbitrary states to initial condition of zero (b). When [ATM*] is zero, Equation (6) represents a pure decaying problem

the reaching of a certain threshold by means of increasing the steady state level of [P53DINP1]. The value of $\varepsilon$ directly determines how fast [P53DINP1] will reach the steady state for a fixed $\beta$ value.

\section{Negative Feedback Loop Naturally Accumulates over Oscillations as a Counting Mechanism}

An important property of a simple negative feedback loop is that it does not decay from an arbitrary state to an initial condition. At the same time, it reaches that arbitrary state if the latter state is smaller than the steady state (Figure 4). To make this point, we have solved the below equation for different arbitrary states of $T_{i}$.

$$
\begin{aligned}
\frac{d[P 53 D I N P 1]}{d t} & =\varepsilon\left(\left[A T M^{*}\right]\right. \\
& -\beta[P 53 D I N P 1])
\end{aligned}
$$


Subject to:

If $t<T_{i}$ then $\left[\right.$ ATM $\left.M^{*}\right]=10$

If $t>T_{i}$ then $\left[A T M^{*}\right]=0$

Where $T_{i} \in\{35,50,100,150\}, \varepsilon=0.02$, and $\beta=1$

If we look at Figure $4 a, b$, it reaches the state of $T_{1}=30$ of [P53DINP1] (which is around 4.5) at 30 hours, however it does not decay to the initial condition of zero at the same time. In fact, there is a large difference. In the case of $T_{4}=150$, the difference between the decay and reach time is smaller since that arbitrary state $\left(T_{4}\right)$ is closer to the steady state.

In order to demonstrate this we take [ATM*] as a sine wave and solve the below equation:

$$
\begin{aligned}
\frac{d[P 53 D I N P 1]}{d t} & =\varepsilon\left(\left[A T M^{*}\right]\right. \\
& -\beta[P 53 D I N P 1])
\end{aligned}
$$

where

$\left[A T M^{*}\right]=5+5 \sin (2 \pi 0.1 t)$

Figure 5 demonstrates that a negative feedback loop can be used as a counting mechanism. It counts the pulses, and after passing a threshold the target network can change the mode. Herein $\varepsilon$ determines how fast [P53DINP1] will move towards the threshold while $\varepsilon \beta$ determines how fast it will decay. Since $\beta$ determines the steady state value, with a proper guess we guarantee that [P53DINP1] will eventually pass the threshold and with $\varepsilon$ we may tune the number of counts until the threshold.

\section{A Compact Motif Capable of Exhibiting Two-phase Dynamics}

Several motifs have been proposed in the literature, such as oscillator motifs and switch motifs $[26,27]$. These simple motifs are the building blocks of more complex gene regulatory systems. In this paper, we introduce a new motif depicted in Figure 6. It consists of i) a bistable switch (ATM), ii) a fast negative feedback loop (Wip1) responsible for the oscillations together with ATM, and iii) a slow negative feedback loop (P53DINP1) responsible for counting the pulses to give the decision of apoptosis. This compact motif with only essential components models the response mechanism of p53 network to DSBs.

In this compact motif, oscillations occur due to the interaction between bistable switch of ATM and negative feedback of Wip1 upon the stimulus [19]. The high level is observed when P53DINP1 level accumulates above a certain threshold value which distracts the feedback loop of Wip1. In this case, the system is left only with bistable ATM dynamics, thus ATM level switches to high state and stays there (Figure 7). Low level is observed due to the fact that bistable ATM do not receive input stimulus thus staying at low level. This notion emphasizes

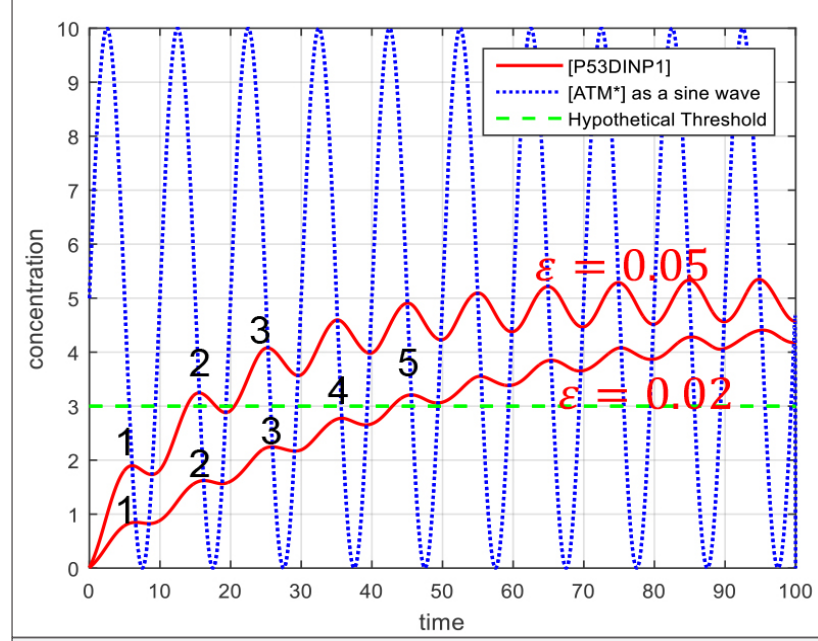

Figure 5. Tuning the number of pulses with different $\varepsilon$ guaranteed that $\beta$ is chosen such that the trajectories will eventually pass the threshold

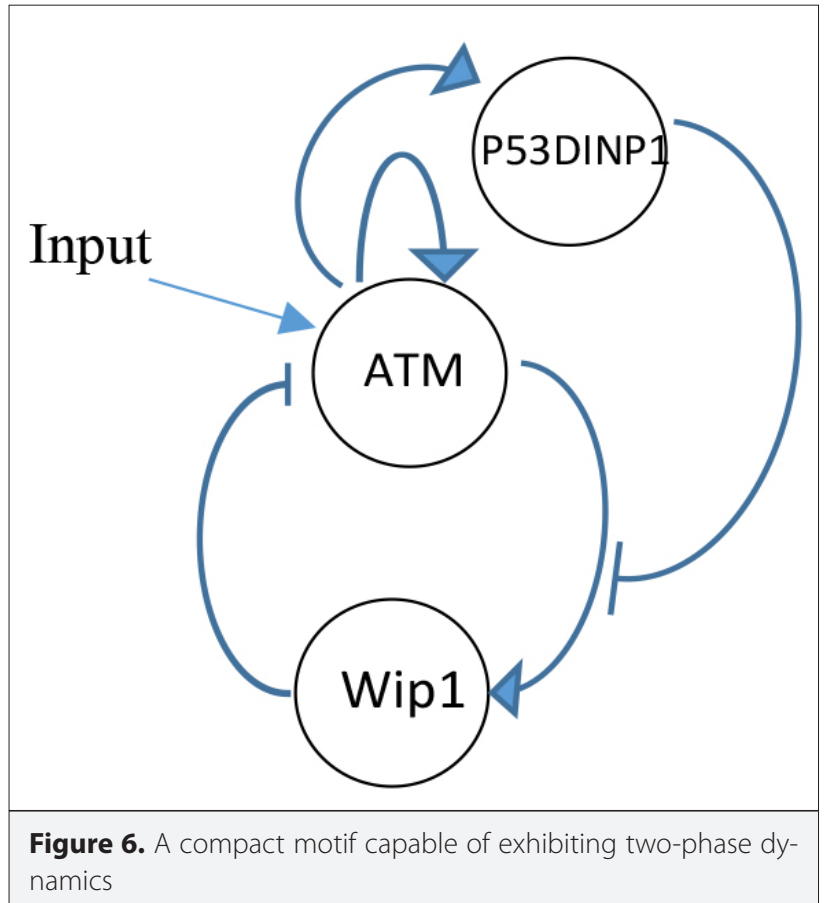

the importance of ATM characteristics on the formation of response to DSBs. That is, other variables in the motif control the ATM dynamics. More specifically, i) Wip1 feedback loop allows for oscillations by frustrating bistable ATM [19], ii) P53DINP1 makes ATM switch from oscillations to high level by distracting Wip1 feedback loop (Figure 8), and iii) the extinction of stimulus make ATM switch to low level.

The compact motif capable of exhibiting two-phase dynamics is a new one: We have shown that a negative feedback can be used as both a counting mechanism and as a switch. Similar to 


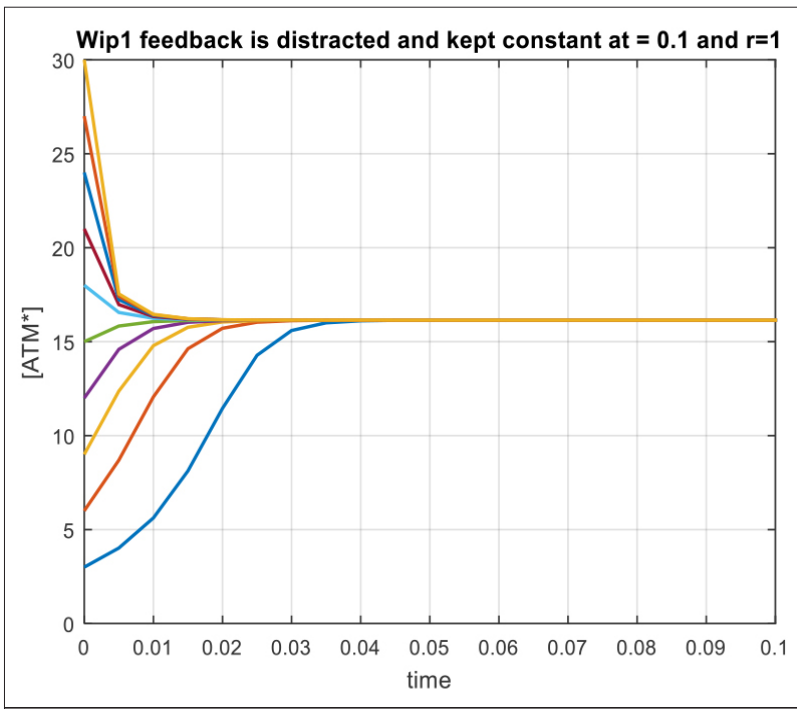

Figure 7. High level of [ATM* ${ }^{*}$ is observed when Wip1 feedback loop is distracted by [P53DINP1]. Only Equation (1) is solved with [Wip1] as constant of 0.1 and $r=1$ for different initial conditions of [ATM*]

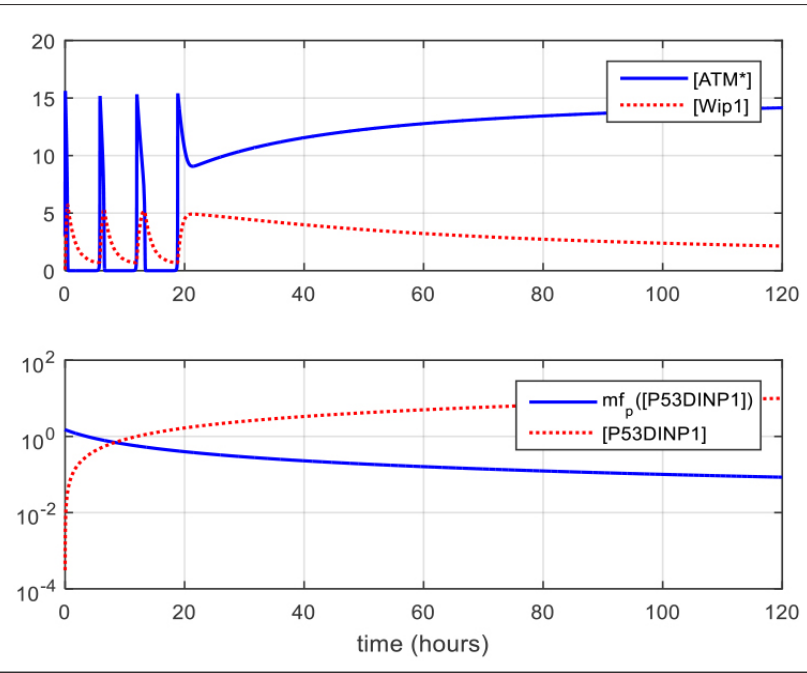

Figure 8. Equations (1) and (2) are solved for $[P 53 D / N P 1]=t / 12$, where $t$ is time. As [P53DINP1] increases with time, the function $m_{f}$ ([P53DINP1]) decreases eventually distracting Wip1 feedback loop and apoptosis is initiated

our study, a generic OATGS, which is able to exhibit transient oscillations, was studied in [28]. However, our mechanism is substantially different from theirs. For instance, [28] proposed a 7-D model that only allows the transitions from low level equilibrium to oscillation and oscillation to low level equilibrium. That is, as the input stimulus persists for a long time, the oscillator causes accumulation of a protein which then shuts off the oscillator to low value. In contrast, in our case, accumulating protein P53DINP1 shuts off the oscillator to a high level if the input stimulus persists for a long time. So, our proposed model, although with lower dimensionality, is richer in dynamics: the proposed model allows for the transitions from low level to os-
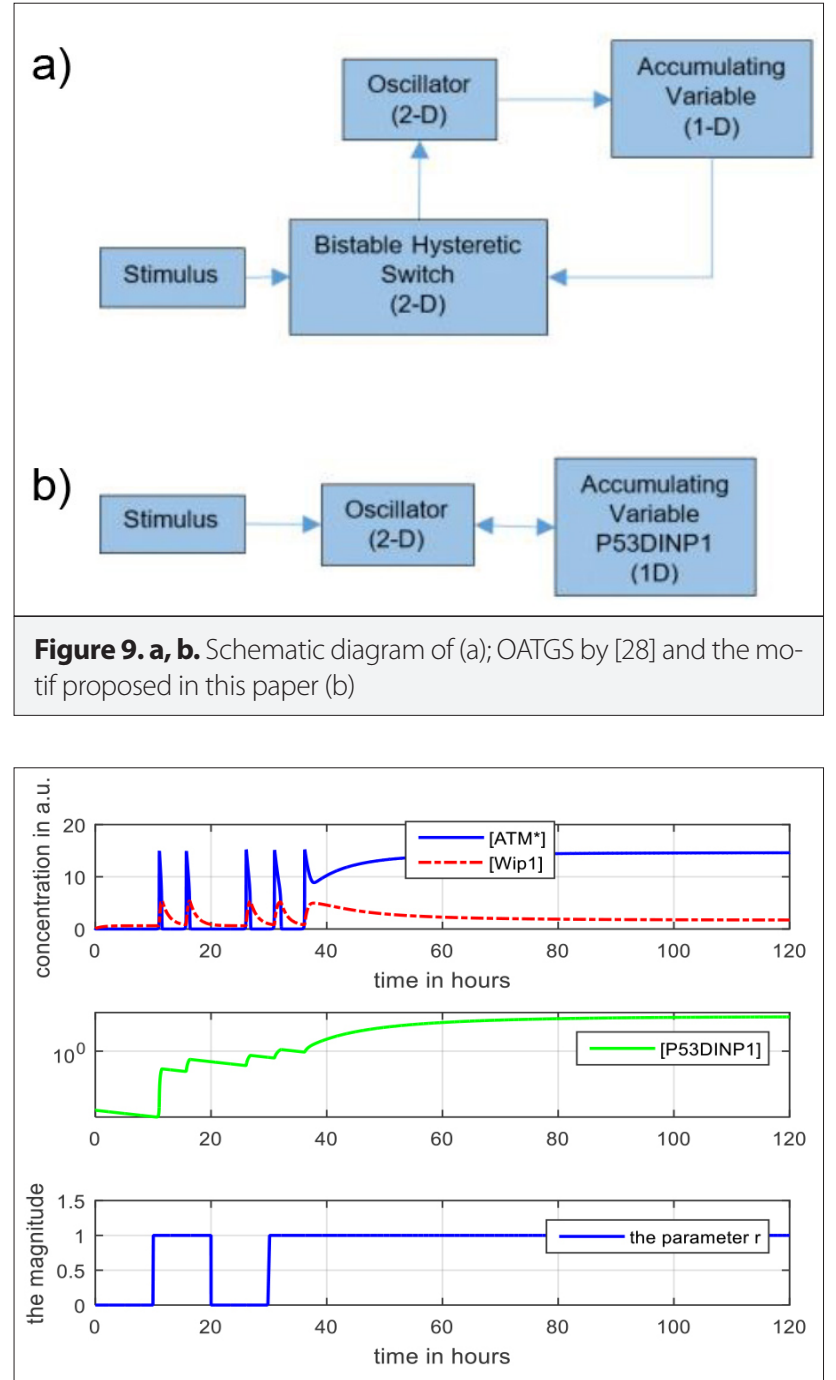

Figure 10. Demonstration of the coincidence detection property of the proposed motif

cillation, oscillation to low level and oscillation to high levels, which are relevant to two-phase dynamics. Both the schematic diagram from [28] and ours are sketched in Figure 9.

\section{Coincidence Detection}

An interesting property of the proposed model is its ability of coincidence detection [26]. Coincidence detection is the phenomenon that describes the support from the past signal to the current signal. We re-simulate Figure 2 with more adjacent pulses of DNA damage as given in Figure 10. This time, apoptosis is observed after 3 pulses as opposed to 4 pulses of [ATM*]. This is due to the simple fact that between time interval 20 and 30 there is not enough time for [P53DINP1] to drop to basal level. So, if new DSBs occur before the cell fully gets rid of the signs of a prior DNA damage, then the cell takes the apoptosis decision earlier than normal. This may give the organism survival advantage, since frequent DNA damage in the cell may be 
a signal for some serious failure in the repair mechanism or in some biological functions. Thus, the cell takes the decision of apoptosis quickly.

\section{Conclusion}

In our previous studies, we have emphasized the importance of re-considering p53 network as an integrated system consisting of an oscillator and other subsystems that control this oscillator to contribute to cell fate [18-20]. In this direction, this paper has introduced a novel subsystem that informs the duration of the repair activity to $\mathrm{p} 53$ network oscillator system to determine cell fate. A negative feedback can provide an accumulating variable which can be used as a pulse counting mechanism to keep the information about the duration of oscillations. The resulting modular system consists of a minimum number of components: two variables for oscillations and one for keeping time. As new findings are revealed from p53 network experiments, the proposed mathematical models can be extended and used modularly. Although we investigate the specific case of the count and decide a mechanism for p53 network, the model can be applied to other gene regulatory networks where the decision is based on an accumulating variable.

\section{Peer-review: Externally peer-reviewed.}

Conflict of Interest: The Authors have no conflicts of interest to declare.

Financial Disclosure: The authors declared that this study has received no financial support.

\section{References}

1. F. Murray-Zmijewski, E. A. Slee, X. Lu, "A complex barcode underlies the heterogeneous response of p53 to stress," Nat Rev Mol Cell Biol, vol. 9, no. 9, pp. 702-712, 2008.

2. D. Michael, M. Oren, "The p53-Mdm2 module and the ubiquitin system," Semin Cancer Biol, vol. 13, no. 1, pp. 49-58, 2003.

3. G. Lahav, N. Rosenfeld, A. Sigal, N. Geva-Zatorsky, A. J. Levine, M. B. Elowitz, U. Alon, "Dynamics of the p53-Mdm2 feedback loop in individual cells," Nat Genet, vol. 36, no. 2, pp. 147-150, 2004.

4. E. Batchelor, A. Loewer, C. Mock, G. Lahav, "Stimulus-dependent dynamics of p53 in single cells," Mol Syst Biol, vol. 7, no. 1, p. 488, 2011.

5. E. Batchelor, A. Loewer, G. Lahav, "The ups and downs of p53: Understanding protein dynamics in single cells", Nat Rev Cancer, vol. 9, no. 5, pp. 371-377, 2009.

6. E. Batchelor, C. S. Mock, I. Bhan, A. Loewer, G. Lahav, "Recurrent initiation: a mechanism for triggering $\mathrm{p} 53$ pulses in response to DNA damage", Mol Cell, vol. 30, no. 3, pp. 277-289, 2008.

7. J. E. Toettcher, A. Loewer, G. J. Ostheimer, M. B. Yaffe, B. Tidor, G. Lahav, "Distinct mechanisms act in concert to mediate cell cycle arrest", Proc Natl Acad Sci, vol. 106, no. 3, pp. 785-790, 2009.

8. G. Lahav, "The strength of indecisiveness: oscillatory behavior for better cell fate determination", Send to SCi STKE, vol. 2004, no. 264, pp. pe55, 2004.

9. F. Essmann, I. H. Engels, G. Totzke, K. Schulze-Osthoff, R. U. Jänicke, "Apoptosis resistance of MCF-7 breast carcinoma cells to ionizing radiation is independent of $\mathrm{p} 53$ and cell cycle control but caused by the lack of caspase-3 and a caffeine-inhibitable event", Cancer Res, vol. 64, no. 19, pp. 7065-7072, 2004.

10. N. D. Marchenko, A. Zaika, U. M. Moll, "Death signal-induced localization of p53 protein to mitochondria a potential role in apoptotic signaling", J Biol Chem, vol. 275, no. 21, pp. 16202-16212, 2000.

11. M. Mihara, S. Erster, A. Zaika, O. Petrenko, T. Chittenden, P. Pancoska, U. M. Moll, "p53 has a direct apoptogenic role at the mitochondria", Mol Cell, vol. 11, no. 3, pp. 577-590, 2003.

12. S. Shreeram, W. K. Hee, O. N. Demidov, C. Kek, H. Yamaguchi, A. J. Fornace, D. V. Bulavin, "Regulation of ATM/P53-dependent suppression of myc-induced lymphomas by Wip1 phosphatase", J Exp Med, vol. 203, no. 13, pp. 2793-2799, 2006.

13. S. Shreeram, O. Demidov, W. Hee, H. Yamaguchi, N. Onishi, C. Kek, O. Timofeev, C. Dudgeon, A. Fornace, C. Anderson, Y. Minami, E. Appella, D. V. Bulavin, "Wip1 phosphatase modulates ATM-dependent signaling pathways", Mol cell, vol. 23, no. 5, pp. 757-764, 2006.

14. Y. Darlington, T. Nguyen, S. H. Moon, A. Herron, P. Rao, C. Zhu, X. Lu and L. A. Donehower, "Absence of Wip1 partially rescues Atm deficiency phenotypes in mice", Oncogene, vol. 31, no. 9, pp. 1155-1165, 2012.

15. Y. Xia, P. Ongusaha, S. W. Lee, Y. C. Liou, "Loss of Wip1 sensitizes cells to stress-and DNA damage-induced apoptosis", J Biol Chem, vol. 284, no. 26, pp. 17428-17437, 2009.

16. T. Sun, J. Cui, “Dynamics of P53 in response to DNA damage: Mathematical modeling and perspective", Prog Biophys Mol Biol, vol. 119, no. 2, pp. 175-182, 2015.

17. X. P. Zhang, F. Liu, W. Wang, "Two-phase dynamics of p53 in the DNA damage response", Proc Natl Acad Sci USA, vol. 108, no. 22, pp. 8990-8995, 2011.

18. G. Demirkıran, G. Kalaycı Demir, C. Güzeliș, "A 2-dimensional Reduced Oscillator Model with Rational Nonlinearities for p53 Dynamics", $10^{\text {th }}$ International Conference on Electrical and Electronics Engineering (ELECO), 2017, pp. 593-597.

19. G. Demirkıran, G. Kalaycı Demir, C. Güzeliş, "Revealing Determinants of Two-Phase Dynamics of P53 Network under Gamma Irradiation Based On A Reduced 2-D Relaxation Oscillator Model", IET Syst Biol, vol. 12, no. 1, pp. 26-38, 2018.

20. G. Demirkıran, G. Demir and C. Güzeliş, “A 2-Dimensional Model of Polynomial Type for Oscillatory ATM-Wip1 dynamics in p53 network", $10^{\text {th }}$ International Conference on Electrical and Electronics Engineering (ELECO), 2017, pp. 598-601.

21. L. Ma, J. Wagner, J. J. Rice, W. Hu, A. J. Levine, G. A. Stolovitzky., "A plausible model for the digital response of p53 to DNA damage," Proc Natl Acad Sci USA, vol. 102, no. 40, pp. 14266-14271, 2005.

22. T. Uziel, Y. Lerenthal, L. Moyal, Y. Andegeko, L. Mittelman, Y. Shiloh, "Requirement of the MRN complex for ATM activation by DNA damage", EMBO J, vol. 22, no. 20, pp. 5612-5621, 2003.

23. K. Mouri, J. C. Nacher, T. Akutsu, "A mathematical model for the detection mechanism of DNA double-strand breaks depending on autophosphorylation of ATM", PLoS One, vol. 4, no. 4, p. e5131, 2009.

24. N. Avcu, G. Kalaycı Demir, F. Pekergin, H. Alyürük, L. Çavaş and C. Güzeliş, "Discriminant-based bistability analysis of a TMG-induced lac operon model supported with boundedness and local stability results", Turkish Journal of Electrical Engineering \& Computer Sciences, pp. 719-732, 2016.

25. N. Avcu, H. Alyürük, G. Kalaycı Demir, F. Pekergin, L. Cavas, C. Güzeliş., "Determining the bistability parameter ranges of artificially induced lac operon using the root locus method", Comput Biol Med, pp. 75-91, 2015.

26. U. Alon, An Introduction to Systems Biology: Design Principles of Biological Circuits, Boca Raton: CRC Press, 2006. 
27. J. J. Tyson, K. C. Chen, B. Novak, "Sniffers, buzzers, toggles and blinkers: dynamics of regulatory and signaling pathways in the cell," Curr Opinn Cell Biol, vol. 15, no. 2, pp. 221-231, 2003.
28. Q. He, Z. Liu, "Investigation of oscillation accumulation triggered genetic switch in gene regulatory networks," Journal of theoretical biology, vol. 353, no. 2014, pp. 61-66, 2014.

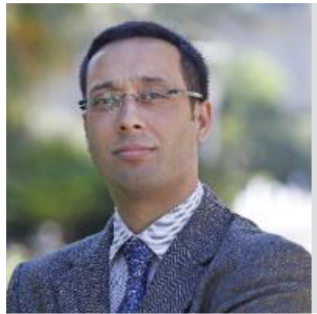

Gökhan Demirkıran received his B.S., M.Sc. and Ph. D. Degree from the Department of Electrical and Electronics Engineering, Dokuz Eylül University. He worked as a research assistant in the Department of Electrical and Electronics Engineering, Yaşar University between the years 2009-2016. He is currently employed as a lecturer in the same department since 2016. His current research interests include systems biology, mathematical modeling, and neural networks.

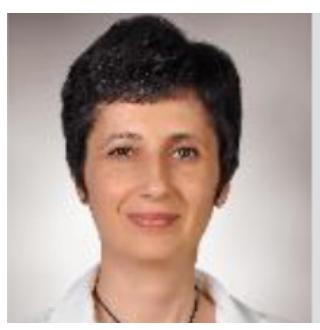

Güleser Kalaycı Demir received her M.Sc. and Ph.D. degrees in Electrical and Electronics Engineering from Dokuz Eylül University (DEU), Izmir, Turkey, in 1996 and 2001, respectively. She worked in the Department of Computer Science and Engineering at University of Minnesota as a Visiting Researcher. She is currently an Assistant Professor in the Department of Electrical and Electronics Engineering at the DEU. Her research interests include control, pattern recognition, systems biology, and machine vision.

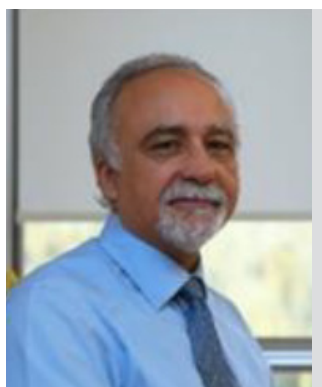

Cüneyt Güzeliş received the B.Sc., M.Sc., and Ph.D. degrees in electrical engineering from İstanbul Technical University, İstanbul, Turkey, in 1981, 1984, and 1988, respectively. He was with İstanbul Technical University from 1982 to 2000 where he became a full professor. He worked between 1989 and 1991 in the Department of Electrical Engineering and Computer Sciences, University of California, Berkeley, California, as a visiting researcher and lecturer. He was with the Department of Electrical and Electronics Engineering from 2000 to 2011 at Dokuz Eylül University, İzmir, Turkey. He was with İzmir University of Economics, Faculty of Engineering and Computer Sciences, Department of Electrical and Electronics Engineering from 2011 to 2015. $\mathrm{He}$ is currently working in Yaşar University, Faculty of Engineering, Department of Electrical and Electronics Engineering. His research interests include artificial neural networks, biomedical signal and image processing, nonlinear circuits-systems, and control, systems biology, and educational systems. 